\title{
Diagnosis and management of food allergy
}

\author{
Elissa M. Abrams MD, Scott H. Sicherer MD
}

$\mathrm{F}$ ood allergy is estimated to affect $2 \%-10 \%$ of the population, with higher rates among children than among adults. ${ }^{1}$ The prevalence of childhood food allergies has apparently increased, with the US Centers for Disease Control and Prevention reporting an increase from $3.4 \%$ to $5.1 \%$ between 1997 and 2011 in the United States. ${ }^{2}$

Food allergy is associated with a high morbidity, affecting day-to-day quality of life. ${ }^{3}$ A study involving 46 families of children with peanut allergy reported decreased overall quality of life $(p<0.05)$ and reduced quality of life within school $(p<0.01)$, as well as increased separation anxiety $(p<0.05){ }^{4}$

Self-reported rates of food allergy are much higher than the true prevalence. ${ }^{5}$ For example, a population-based cohort study involving 3623 children found that $35 \%$ of parents perceived food reactions in their children. ${ }^{5}$ Unnecessary food avoidance could have unintended consequences, including nutritional deficiency. ${ }^{3}$ Physicians play a pivotal role in ensuring food allergy is properly diagnosed and managed, both to ensure avoidance of causal foods and to prevent unnecessary food avoidance.

In this review, we focus on immunoglobulin E (IgE)-mediated food allergy and address key issues in its diagnosis and management, offering advice on how clinicians can avoid common pitfalls and improve patient care. Our search strategy is summarized in Box 1.

\section{Box 1: Evidence used in this review}

After identifying common issues facing primary care providers in the diagnosis and management of food allergies, we used recent Canadian, American and international practice parameters and guidelines as a primary basis to inform this review, supplemented with a search for systematic reviews for supporting information. Additional clinical points and examples are based on reviews, cohort and case-control studies, and surveys. We restricted our search to English-language articles. Where possible, we selected the most recent articles and the articles with the most robust level of evidence. We reviewed more than 100 citations, of which 54 are included in this review.

\section{What is food allergy and how does it present?}

Food allergy is defined as "an adverse health effect arising from a specific immune response that occurs reproducibly on exposure to a given food." ${ }^{3}$ In contrast, other nonimmune adverse reactions may occur from foods and should be differentiated from food allergy. These include intolerance (e.g., lactose intolerance that could be differentiated by history or hydrogen breath test), illness following ingestion of contaminated food and pharmacologic effects (e.g., caffeine). ${ }^{6}$ Food allergy can be classified according to the nature of the immune response as IgE-mediated, non-IgE-mediated (cell mediated) or mixed IgEand non-IgE-mediated (Table 1). ${ }^{6}$

IgE-mediated food reactions occur when a food allergen binds allergen-specific IgE present on mast cells and basophils, which leads to the release of multiple mediators such as histamine. The reactions occur rapidly (within two hours) and may include one or more of cutaneous, respiratory, gastrointestinal or cardiovascular symptoms (Table 2). ${ }^{6}$ Symptoms resolve within hours and occur reproducibly with repeat exposure to the culprit food. An unusual exception to the otherwise rapid onset of symptoms is one form of allergy to red meat attributed to $\operatorname{IgE}$ antibodies against the sugar moiety galactose- $\alpha-1,3-$ galactose, where symptom onset is delayed two to six hours. ${ }^{7}$

Cutaneous symptoms are by far the most common. ${ }^{3}$ Although respiratory symptoms are

\section{KEY POINTS}

- Food allergy has a characteristic and reproducible clinical presentation.

- Testing for food allergy is highly sensitive, but it has a low positive predictive value and often identifies clinically insignificant sensitization.

- The only life-saving treatment for food-induced anaphylaxis is epinephrine.

- Egg allergy is not a contraindication to influenza or measles-mumpsrubella vaccination.

- Allergies to milk, egg, soy and wheat will resolve during childhood in most instances; allergies to peanuts, tree nuts and seafood tend to persist. 
often observed during a food allergy reaction, isolated chronic respiratory symptoms (e.g., asthma and rhinitis) are not typically attributable to food allergy. ${ }^{6}$

Headaches, chronic abdominal pain and chronic behavioural symptoms are unlikely to represent food allergy. Food allergy only accounts for about $20 \%$ of all acute urticaria ${ }^{8}$ and is not a likely cause of chronic urticaria (defined as frequent daily hives for more than six weeks). ${ }^{8}$

Pollen-food allergy syndrome, also known as oral allergy syndrome, typically presents with isolated oropharyngeal symptoms (e.g., pruritus and mild angioedema) after ingestion of raw fruit or vegetables. The syndrome is due to protein cross-reactivity between heat-labile proteins in food and in pollens. ${ }^{8}$ Most patients can tolerate the cooked form of the food because the causal protein is degraded by heat. ${ }^{8}$ Common

Table 1: Classification of food allergy ${ }^{6}$

\begin{tabular}{|ll|}
\hline Type of food allergy & \multicolumn{1}{c|}{ Examples } \\
\hline IgE-mediated & $\begin{array}{l}\text { Anaphylaxis, pollen-food allergy syndrome, } \\
\text { contact urticaria }\end{array}$ \\
\hline Non-IgE-mediated & $\begin{array}{l}\text { Food protein-induced enterocolitis syndrome, } \\
\text { food protein-induced proctocolitis }\end{array}$ \\
\hline $\begin{array}{l}\text { Mixed IgE- and } \\
\text { non-IgE-mediated }\end{array}$ & Eosinophilic esophagitis \\
\hline
\end{tabular}

Table 2: Symptoms of IgE-mediated food allergy ${ }^{6}$

\begin{tabular}{|ll|}
\hline Organ system & \multicolumn{1}{c|}{ Examples } \\
\hline Cutaneous & Urticaria, angioedema, flushing, pruritus \\
\hline Upper respiratory & Sneezing, rhinorrhea, congestion, conjunctivitis \\
\hline Lower respiratory & Shortness of breath, wheeze, cough \\
\hline Gastrointestinal & Nausea, vomiting, diarrhea, pain \\
\hline Cardiovascular & Hypotension, syncope \\
\hline Central nervous system & Sense of impending doom \\
\hline
\end{tabular}

Table 3: Clinical presentation of selected non-IgE mediated, and mixed IgE- and non-lgE-mediated food allergies ${ }^{6}$

\begin{tabular}{|ll|}
\hline Disorder & \multicolumn{1}{c|}{ Clinical features } \\
\hline $\begin{array}{l}\text { Food protein-induced } \\
\text { enterocolitis (non-IgE-mediated) }\end{array}$ & $\begin{array}{l}\text { Severe vomiting, lethargy, possible } \\
\text { hypotension or cyanosis, with symptom } \\
\text { onset two hours after ingestion; common } \\
\text { triggers are milk, soy, rice and oats }\end{array}$ \\
\hline $\begin{array}{l}\text { Food protein-induced } \\
\text { proctocolitis (non-lgE-mediated) }\end{array}$ & $\begin{array}{l}\text { Bloody stools in an otherwise healthy } \\
\text { infant; typically occurs in breastfed } \\
\text { infants and is most often related to } \\
\text { maternal ingestion of cow's milk }\end{array}$ \\
\hline $\begin{array}{l}\text { Eosinophilic esophagitis (mixed } \\
\text { IgE and non-lgE mediated) }\end{array}$ & $\begin{array}{l}\text { Aversion to feeding in infants, } \\
\text { abdominal pain, dysphagia, esophageal } \\
\text { food impaction }\end{array}$ \\
\hline
\end{tabular}

associations include birch tree pollen with pitted fruit (e.g., apple, peach and pear) and ragweed pollen with melons. ${ }^{8}$

Contact urticaria presents as isolated urticaria at the site of contact with a food allergen, such as on the hands of a cook upon contact with raw fruit or vegetables. ${ }^{6}$

Anaphylaxis is defined as a "serious allergic reaction that is rapid in onset and may cause death." ${ }^{\prime 3}$ Anaphylaxis usually involves a combination of symptoms from multiple organ systems (e.g., cutaneous, respiratory, gastrointestinal and cardiovascular). Food-dependent exercise-induced anaphylaxis is a particular form of anaphylaxis that requires the temporal combination of exercise and ingestion of a trigger food. ${ }^{6}$ The reaction does not occur after ingestion of the food in the absence of exercise. ${ }^{6}$ The pathophysiology is uncertain but may involve increased systemic absorption of intact food allergens during exercise. Wheat, shellfish and celery are common triggers of food-dependent exercise-induced anaphylaxis. ${ }^{9}$

Table 3 describes common clinical features of several types of non-IgE-mediated and mixed IgE- and non-IgE-mediated food allergies. ${ }^{6}$ Although the focus of our review is on IgEmediated food allergy, it is important to differentiate this from the other types of food allergy. In general, non-IgE-mediated food allergy presents with delayed or chronic, isolated gastrointestinal symptoms. For example, eosinophilic esophagitis presents with predominantly gastrointestinal symptoms that vary by age. ${ }^{6}$ In infants and children, it presents with aversion to feeding, abdominal pain and emesis. In adolescents and adults, it presents predominantly with food impaction and dysphagia. Patients with eosinophilic esophagitis are often sensitized to food allergens. ${ }^{6}$

\section{Is there a genetic disposition to food allergy?}

Both genetic disposition and environmental influences predispose to food allergy. In a study involving 58 pairs of twins, the concordance rate of peanut allergy was significantly higher among monozygotic than among dizygotic twins $(64.3 \%$ v. $6.8 \% ; p<0.0001)$, with an estimated peanut allergy heritability of $81.6 \% .{ }^{10}$ Currently, the specific genetic mutations that confer risk for food allergy are being investigated. In a recent Dutch cohort, two single nucleotide polymorphisms were significantly associated with the development of cow's milk allergy ( $p=0.002$ and 0.038 ) in 36 children. ${ }^{11}$

The apparent increase in the prevalence of food allergy over the past decades cannot be 
explained solely by genetics. There are also several environmental factors that are thought to contribute to food allergy and may partially explain the rise in prevalence. These factors include the "hygiene hypothesis" (which suggests that changes in the microbiome have altered immune responses), the effect of vitamin D deficiency on the immune response, and the notion that delaying ingestion of potent allergens may, especially in allergy-prone infants with atopic dermatitis, result in sensitization by nonoral routes (i.e., sensitizing skin exposure without the benefit of developing tolerance through oral exposure). ${ }^{12} \mathrm{~A}$ recent randomized prospective trial addressed the hypothesis that earlier introduction of an allergen, in this case peanuts, may prevent allergy in high-risk infants. Among infants aged 4-11 months at enrolment who had severe atopic dermatitis or egg allergy, a risk reduction of $86.1 \%$ for peanut allergy at five years of age was reported among infants with early versus late peanut introduction. ${ }^{13}$

\section{How is food allergy diagnosed?}

The approach to diagnosis begins with the patient's medical history, which may identify food allergy as a possible cause of symptoms, provide details suggesting whether the reaction is IgE-mediated and determine the likely culprit allergen(s) (Table 4). Once the suspect food allergen has been identified, first-line diagnostic testing consists of skin testing or measurement of serum food-specific IgE levels, or both.
Serum food-specific IgE measurement identifies IgE antibodies to a food (i.e., sensitization). ${ }^{6,18}$ For skin testing, allergen is introduced into the epidermis using extracts and a device to scratch or puncture the skin. The presence of a wheal and flare indicates sensitization. ${ }^{19}$ Both forms of testing are highly sensitive in the diagnosis of IgEmediated food allergy. The sensitivity is greater than $90 \%$ for skin testing and $70 \%-90 \%$ for serum food-specific IgE measurement. ${ }^{8,18,20}$ Wheal size and IgE level are associated with likelihood of allergy, but neither correlates well with reaction severity. ${ }^{21}$

Skin testing has benefits of lower cost and immediate results. ${ }^{18,21}$ Serum food-specific IgE measurement is more widely available in primary care and may provide an improved ability to address changes in results over time. Depending on the allergen and aspects of test development (e.g., extraction process, manufacture of reagents), one test or another may offer more sensitivity.

Diagnostic testing should be done only in the context of a convincing clinical history. The specificity of both tests is less than $50 \% .{ }^{14}$ Sensitization often does not equate to clinical allergy and can lead to unnecessary food avoidance. ${ }^{21}$ In a recent retrospective chart review of 125 children, $80 \%-100 \%$ of foods avoided because of a positive skin test or serum food-specific IgE test result were avoided unnecessarily and could be reintroduced into the patients' diets. ${ }^{22}$ Testing a prespecified "panel" without attention to the medical history and an understanding of the epi-

Table 4: Useful components of the medical history

Was one of the most common allergenic foods ingested within 2 hours before the onset of the reaction?

Had the suspect food been ingested previously?

Had there been prior reactions?

What is the age of onset?

What was the mode of preparation?

Were there augmenting factors?

What symptoms were experienced by the patient?
A few foods (cow's milk, eggs, peanuts, tree nuts, shellfish, finned fish, wheat and soy) account for most food allergies ${ }^{14}$

A history of repeated tolerance to the food means it is less likely the culprit

Repeated reactions to the same food means it is more likely the culprit

Onset in childhood is mostly likely due to milk, egg, wheat or peanut allergy, whereas onset in adulthood is usually due to nut or shellfish allergy or to pollen-food allergy syndrome ${ }^{8}$

Larger amounts of food are more likely to cause a reaction, although reactions to trace amounts can also occur; a person reactive to whole cow's milk or egg may tolerate lesser amounts of heated forms in baked goods (e.g., cookie, muffin) ${ }^{15,16}$

Exercise, infection, use of medications (e.g., nonsteroidal anti-inflammatory drugs), menstruation and alcohol consumption can increase the likelihood of reactivity or make a reaction more severe ${ }^{17}$

Cutaneous, respiratory, gastrointestinal and cardiovascular symptoms are indicative of an IgE-mediated reaction. An older individual may experience a sense of "doom," whereas a young child may cry, stop playing or become listless 
demiology and test characteristics can result in mismanagement.

A medically supervised oral food challenge is the diagnostic gold standard. ${ }^{3}$ The oral food challenge is generally conducted by specialists with expertise in food allergy and involves having the patient ingest incremental amounts of a food in a medically supervised environment.

The oral food challenge can be open, singleblind or a double-blind placebo-controlled challenge. In an open challenge, both the physician and the patient are aware of the food being ingested. In a single-blind challenge, the patient is unaware, and in a double-blind placebo-controlled challenge, both the physician and the patient are unaware of the food being ingested. Although the double-blind placebo-controlled challenge is the most specific test, it is time-consuming and less commonly used in clinical practice. ${ }^{18}$

The oral food challenge is a standard accepted procedure and is considered safe when undertaken by experienced personnel. However, it is time-consuming and allergic reactions, including anaphylaxis, may be induced. In a retrospective chart review of more than 700 food challenges in one clinic, $19 \%$ of patients reacted, including $2 \%$ who required epinephrine treatment. ${ }^{23}$

Component-resolved diagnostics is an emerging tool in the diagnosis of food allergy. It measures serum IgE levels against individual allergenic proteins within a food rather than a mixture of allergens. ${ }^{24}$ Reactivity of IgE against specific components may be related to the likelihood of clinical allergy. For example, patients with a peanut allergy sensitized to stable peanut proteins (e.g., Ara h 2) have a higher likelihood of reactivity than those solely sensitized to labile proteins (e.g., Ara h 8). ${ }^{24}$ In a study involving 144 children sensitized solely to Ara h 8, only 1 child had a reaction upon ingestion. ${ }^{25}$

Food-specific immunoglobulin G (IgG) testing is being increasingly used to identify food "sensitivities." This testing has not been validated nor supported by research. In fact, foodspecific IgG is to be expected, marking the presence of exposure and tolerance to a food. The Canadian Society of Allergy and Clinical Immunology released a position statement strongly discouraging the use of IgG testing to identify an adverse food reaction, echoing a similar sentiment expressed by the American Academy of Allergy, Asthma and Immunology. ${ }^{26,27}$ Additional tests that are not recommended include applied kinesiology and electrodermal testing. ${ }^{3}$

Until component-resolved diagnostics has been refined, skin testing and serum foodspecific IgE measurement remain the first-line tools in the evaluation of food allergy. Both have strong diagnostic utility if correlated with clinical history. There is no standardized step-wise approach to testing. Skin testing, serum foodspecific IgE testing or both may be used to evaluate IgE-mediated food allergies. However, they should not be relied on as the sole means of diagnosis, because the clinical history, and possibly the performance of medically supervised oral food challenges, are key additional components of the diagnostic regimen.

\section{What treatment is life-saving in food-induced anaphylaxis?}

Multiple guidelines recommend intramuscular epinephrine as the first-line treatment of anaphylaxis. ${ }^{3,28}$ Epinephrine's beneficial mechanisms of action include decreasing laryngeal edema, vasoconstriction (alleviating hypotension), increasing inotropic and chronotropic effects, bronchodilation and reducing release of inflammatory mediators from mast cells. . $^{3,28,29}$

Delay or lack of epinephrine administration has been associated with increased morbidity and mortality. ${ }^{30,31}$ Prompt administration of epinephrine was associated with lower rates of hospital admission in a study of 384 emergency department visits for food-induced anaphylaxis (17\% v. $43 \%$ among patients given epinephrine late [in the emergency department]; $p<0.001){ }^{32}$ Death from food-induced anaphylaxis can occur quickly (within 30-60 min), ${ }^{24}$ and hence prompt treatment with epinephrine is required. ${ }^{3,28}$

Epinephrine autoinjectors are often underused. A survey of 1885 people who survived anaphylactic reactions found that autoinjectors were used in only $27 \%$ of the episodes. Of those who did not use an epinephrine autoinjector, $38 \%$ reported taking an antihistamine instead and $28 \%$ had no epinephrine prescription. ${ }^{33}$

Patients with potentially life-threatening food allergy should be prescribed an epinephrine autoinjector and educated in its use. Epinephrine autoinjectors are available in two fixed doses: $0.15 \mathrm{mg}$ and $0.30 \mathrm{mg}$. Guidelines suggest using the 0.30 -mg dose if the person's weight is about $25 \mathrm{~kg}$ or higher. ${ }^{3,34}$

It is important to educate patients that epinephrine is both effective and safe. Although transient adverse effects (e.g., tremor, palpitations and anxiety) are common, more serious adverse effects (e.g., hypertension and angina) are rare and are usually attributable to overdose or incorrect administration caused by intravenous dosing rather than use of autoinjectors. ${ }^{35} \mathrm{~A}$ cohort study involving 573 patients (301 of whom received epinephrine) noted much higher 
rates of cardiovascular complications with intravenous than with intramuscular epinephrine administration (10\% v. $1.3 \% ; p=0.006) .{ }^{35}$ Of note, cardiovascular disease is not a contraindication to epinephrine administration. ${ }^{28}$

Pending evaluation by an allergist, patients and their families may also be directed to websites for further information and support (Box 2).

Adjunct treatments may include the use of $\mathrm{H}_{1}$-antihistamines, bronchodilators and corticosteroids. None of these interventions is lifesaving, and none should be used in place of epinephrine when treating anaphylaxis. ${ }^{3,28}$

In addition to ensuring that patients at risk of life-threatening food allergy have an epinephrine autoinjector, there are patient-specific factors that can increase anaphylaxis severity and risk of death that should also be addressed. These include age (e.g., risk-taking behaviours of adolescents), comorbid conditions (e.g., asthma and cardiovascular disease) and concurrent medication use (e.g., $\beta$-blockers, which make it more difficult to treat anaphylaxis). ${ }^{29}$ Prudent management of comorbid conditions, regular review of medications and routine reinforcement of anaphylaxis treatment is recommended. ${ }^{29}$

\section{Does allergy to a food require avoidance of related foods?}

Rates of cross-reactivity vary extensively among the common food allergens (Table 5). ${ }^{36}$ Patients allergic to shellfish or finned fish (e.g., tuna, salmon and cod) may need to avoid that entire food group because of high rates of cross-reactivity. ${ }^{6,36}$ However, it is possible for individuals to have isolated food allergy as well, for example only to swordfish. Even among foods with high rates of cross-reactivity, there are nuances. For example, walnuts are related to pecans, cashews to pistachios, and almonds to hazelnuts. ${ }^{6,36}$ If there is a desire to eat a food from the same food group as the implicated allergen, diagnostic evaluation (e.g., skin testing or oral food challenge, or both) could be considered in the absence of prior tolerance. ${ }^{6,36}$ When selecting foods in this manner, patients

\section{Box 2: Resources for patients}

- Food Allergy Canada: www.foodallergycanada.ca

- Food Allergy Research and Education: www.foodallergy.org

- Anaphylaxis Emergency Action Plan: https://www.aaaai.org/aaaai/media/ medialibrary/pdf\%20documents/libraries/ anaphylaxis-emergency-action-plan.pdf must take care to avoid cross-contact or misidentification of the allergens. ${ }^{36}$ For example, a walnut brownie may appear safe for a person allergic to cashews and Brazil nuts, but it could cause a reaction if it contains or is unintentionally contaminated with other nuts the patient needs to avoid.

Patients with latex allergy may also be crosssensitized to food allergens because of homologous proteins. For example, as many as $35 \%$ of patients with latex allergy may be sensitized to fruits such as kiwi, avocado and banana, and as many as $11 \%$ of patients with these fruit allergies may be sensitized to latex. ${ }^{36}$

\section{What is the prognosis of food allergy?}

Allergies to milk, egg, soy and wheat will resolve during childhood in most instances, ${ }^{3}$ although age of resolution varies ${ }^{3,37-42}$ (Table 6). In contrast, allergies to peanuts, tree nuts, finned fish and shellfish tend to persist. ${ }^{3}$

The rate of resolution varies depending on the presence of other atopic disease, ${ }^{37-40}$ the serum IgE level or size of wheal on skin testing, ${ }^{37-46}$ and the characteristics of the initial reaction..$^{38}$

The prognosis of egg and milk allergies may

Table 5: Rates of clinical cross-reactivity among food allergens ${ }^{36}$

\begin{tabular}{|ll|}
\hline Food group & \multicolumn{1}{c|}{ Rate of cross-reactivity } \\
\hline Shellfish (e.g., shrimp, lobster) & Other shellfish $75 \%$ \\
\hline Finned fish (e.g., tuna, salmon, cod) & Other finned fish $50 \%$ \\
\hline $\begin{array}{l}\text { Tree nuts (e.g., cashews, walnuts, } \\
\text { pecans) }\end{array}$ & $\begin{array}{l}\text { Other tree nuts } 37 \% \text { (higher for } \\
\text { walnut/pecan and cashew/pistachio) }\end{array}$ \\
\hline Peanut & Other legumes (peas, beans) $5 \%$ \\
\hline Grains (e.g., wheat, barley, oats) & Other grains $20 \%$ \\
\hline Cow's milk & Goat's milk $90 \%$ \\
\hline
\end{tabular}

Table 6: Rates of resolution of common food allergies during childhood

\begin{tabular}{|lcl|}
\hline Food allergy & $\begin{array}{c}\text { Rate of resolution } \\
\text { during childhood, } \%\end{array}$ & \multicolumn{1}{c|}{ Age of resolution } \\
\hline Egg $^{37,38}$ & $49-68$ & $\begin{array}{l}4 \% \text { by } 4 \text { yr, } 12 \% \text { by } 6 \mathrm{yr}, 37 \% \text { by } \\
10 \mathrm{yr}, 68 \% \text { by } 16 \mathrm{yr}^{37}\end{array}$ \\
\hline Cow's milk & $52-79$ & $\begin{array}{l}19 \% \text { by } 4 \mathrm{yr}, 64 \% \text { by } 12 \mathrm{yr}, 79 \% \\
\text { by } 16 \mathrm{yr}^{40}\end{array}$ \\
\hline Soy $^{41}$ & 69 & $\begin{array}{l}25 \% \text { by } 4 \mathrm{yr}, 45 \% \text { by } 6 \mathrm{yr}, 69 \% \\
\text { by } 10 \mathrm{yr}^{41}\end{array}$ \\
\hline Wheat ${ }^{42}$ & 65 & $\begin{array}{l}29 \% \text { by } 4 \text { yr, } 56 \% \text { by } 8 \mathrm{yr}, 65 \% \\
\text { by } 12 \mathrm{yr}^{42}\end{array}$ \\
\hline Peanut ${ }^{43-45}$ & $\sim 20$ & insufficient data \\
\hline Tree nut ${ }^{46}$ & 9 & insufficient data \\
\hline
\end{tabular}


be altered by the ingestion of baked products that contain egg or milk ingredients for people who can tolerate these forms. ${ }^{15,16}$ However, some patients may be unable to tolerate the extensively heated forms of these two foods and experience anaphylaxis from them. Patients with egg or milk allergy may be evaluated for potential tolerance to baked egg or milk (by skin testing or serum food-specific IgE measurement, and oral food challenge, as warranted). If tolerant, regular consumption is strongly encouraged.

Yearly re-evaluation with a clinical history or serum food-specific IgE measurement, or both, is often recommended for all food allergens, especially in young children. ${ }^{3}$ The frequency of testing depends on a number of variables, including the age of the child, the specific allergenic food and whether there have been any intervening exposures or reactions. ${ }^{3}$ The frequency can be reduced with age, especially for the allergens that are less commonly outgrown. Routine reevaluation is also an opportunity to review food avoidance and emergency management.

Although prognosis is often favourable, food allergy is a risk factor for other atopic disease. Patients with food allergies are two to four times more likely than those without food allergy to have atopic dermatitis, asthma and rhinitis. ${ }^{3}$ Therefore, it is prudent to monitor for the development of other allergic disease.

\section{Which vaccines are contraindicated in children with egg allergy?}

Influenza and measles-mumps-rubella (MMR) vaccines are not contraindicated in children with egg allergy ${ }^{47}$ and should be administered according to the routine vaccination schedule. ${ }^{48}$

Influenza vaccines are cultured on embryonated chicken eggs, and MMR vaccine is grown in cultures of chick embryo fibroblasts. ${ }^{47}$ However, in 28 studies $(n=4315)$ of influenza vaccination in patients with egg allergy, no serious reactions occurred. ${ }^{49}$ In addition, multiple studies have shown the safety of MMR vaccination in patients with egg allergy. ${ }^{50,51}$ As a result, the Canadian Immunization Guide states that MMR, trivalent and quadrivalent inactivated influenza vaccines can be administered as a single dose without prior testing in patients with egg allergy. ${ }^{48}$

The American guideline on influenza vaccination in patients with egg allergy is slightly different. The American Academy of Allergy, Asthma and Immunology specifies that influenza vaccine should be administered in an allergist's office if the patient's reaction to egg was more than isolated hives. ${ }^{47}$ Both the American and
Canadian guidelines recommend monitoring the patient after vaccination (for 30 minutes and for 15-30 minutes, respectively). ${ }^{47,48}$ Similar to the Canadian guideline, the American guideline states that influenza vaccine can be given as a single dose without prior testing. ${ }^{47}$

Live-attenuated influenza vaccine in the form of a nasal spray is not yet recommended in individuals with egg allergy because its use has not been extensively studied in this population. ${ }^{52}$ One recent study noted no systemic IgE-mediated reactions in 779 children with egg allergy given this form of influenza vaccine.$^{53}$ Further studies are pending (ClinicalTrials.gov NCT01859039 and NCT02111512), and guidelines are likely to change.

As with all vaccines, MMR and influenza vaccines should be administered in a setting where anaphylaxis can be managed. ${ }^{48}$ Patients who have an IgE-mediated reaction to any vaccine should be evaluated by an allergist. ${ }^{47}$

Egg allergy is a relative contraindication to the administration of yellow fever vaccine, tickborne encephalitis vaccine and one type of rabies vaccine (RabAvert), because these vaccines contain larger amounts of egg protein. ${ }^{48}$ If required, evaluation by an allergist is recommended because desensitization protocols have been described. ${ }^{54}$

\section{Conclusion}

Food allergy is prevalent, although self-reported rates are much higher than true prevalence. An understanding of the characteristic clinical presentation can help with diagnosis. Diagnostic testing is highly sensitive and often identifies clinically irrelevant sensitization. Testing therefore must be selected and interpreted in the context of the patient's clinical history. Oral food challenge remains the gold standard in diagnostic testing. Patients with potentially life-threatening food allergy require an epinephrine autoinjector, as well as training on its use. Prognosis varies depending on the food allergen, and food allergy is a risk factor for other atopic diseases.

\section{References}

1. Chafen JJ, Newberry SJ, Riedl MA. Diagnosing and managing common food allergies: a systematic review. JAMA 2010;303: 1848-56.

2. Jackson KD, Howie LD, Akinbami LJ. Trends in allergic conditions among children: United States, 1997-2011. NCHS Data Brief 2013;(121):1-8.

3. NIAID-Sponsored Expert Panel; Boyce JA, Assayed A, Burks AW, et al. Guidelines for the diagnosis and management of food allergy in the United States: report of the NIAID-sponsored expert panel. J Allergy Clin Immunol 2010;126:S1-58.

4. King RM, Knibb RC, Hourihane JO. Impact of peanut allergy on quality of life, stress and anxiety in the family. Allergy 2009; 64:461-8.

5. Eggesbø M, Halvorsen R, Tambs K, et al. Prevalence of parentally perceived adverse reactions to foods in young children. Pediatr Allergy Immunol 1999;10:122-32. 
6. Sampson HA, Aceves S, Bock SA, et al. Food allergy: a practice parameter update - 2014. J Allergy Clin Immunol 2014;134 1016-25.e43.

7. Platts-Mills TA, Schuyler AJ, Hoyt AE, et al. Delayed anaphylaxis involving IgE to galactose-alpha-1,3-galactose. Curr Allergy Asthma Rep 2015;15:12.

8. Sicherer SH, Sampson HA. Food allergy. J Allergy Clin Immunol 2010;125:S116-25.

9. Beaudouin E, Renaudin JM, Morisset M, et al. Food-dependent exercise-induced anaphylaxis - update and current data. Eur Ann Allergy Clin Immunol 2006;38:45-51.

10. Sicherer SH, Furlong TJ, Maes HH, et al. Genetics of peanut allergy: a twin study. J Allergy Clin Immunol 2000;106:53-6.

11. Henneman P, Petrus NC, Venema A, et al. Genetic susceptibility for cow's milk allergy in Dutch children: The start of the allergic march? Clin Transl Allergy 2016;6:7.

12. Allen KJ, Koplin JJ. Prospects for prevention of food allergy. J Allergy Clin Immunol Pract 2016;4:215-20.

13. Du Toit G, Roberts G, Sayre PH, et al. Randomized trial of peanut consumption in infants at risk for peanut allergy. $N$ Engl $J$ Med 2015;372:803-13.

14. Sampson HA. Food allergy. Part 2: diagnosis and management J Allergy Clin Immunol 1999;103:981-9.

15. Leonard SA, Sampson HA, Sicherer SH, et al. Dietary baked egg accelerates resolution of egg allergy in children. J Allergy Clin Immunol 2012;130:473-80.e1.

16. Kim JS, Nowak-Wegrzyn A, Sicherer SH, et al. Dietary baked milk accelerates the resolution of cow's milk allergy in children. J Allergy Clin Immunol 2011;128:125-131.e2.

17. Niggemann B, Beyer K. Factors augmenting allergic reactions. Allergy 2014;69:1582-7.

18. Kattan JD, Sicherer SH. Optimizing the diagnosis of food allergy. Immunol Allergy Clin North Am 2015;35:61-76.

19. Hamilton RG. Clinical laboratory assessment of immediate-type hypersensitivity. J Allergy Clin Immunol 2010;125:S284-96.

20. Cox L, Williams B, Sicherer S, et al. Pearls and pitfalls of allergy diagnostic testing: report from the American College of Allergy, Asthma and Immunology/American Academy of Allergy, Asthma and Immunology Specific IgE Test Task Force. Ann Allergy Asthma Immunol 2008;101:580-92.

21. Lieberman JA, Sicherer SH. Diagnosis of food allergy: epicutaneous skin tests, in vitro tests, and oral food challenge. Curr Allergy Asthma Rep 2011;11:58-64.

22. Fleischer DM, Bock A, Spears GC, et al. Oral food challenges in children with a diagnosis of food allergy. J Pediatr 2011;158:578-583.e1.

23. Lieberman JA, Cox AL, Vitale M, et al. Outcomes of officebased, open food challenges in the management of food allergy. J Allergy Clin Immunol 2011;128:1120-2.

24. Sicherer SH, Sampson HA. Food allergy: epidemiology, pathogenesis, diagnosis and treatment. J Allergy Clin Immunol 2014 133:291-307.

25. Asarnoj A, Nilsson C, Lidholm J, et al. Peanut component Ara h 8 sensitization and tolerance to peanut. J Allergy Clin Immunol 2012;130:468-72

26. Carr S, Chan E, Lavine E, et al. CSACI position statement on the testing of food-specific IgG. Allergy Asthma Clin Immunol 2012;8:12.

27. Bock SA. AAAAI support of the EAACI position paper on IgG4. J Allergy Clin Immunol 2010;125:1410.

28. Simons FE, Arusso LR, Bilò MB, et al. World Allergy Organization anaphylaxis guidelines: summary. J Allergy Clin Immunol 2011;127:587-93.e1-22.

29. Simons FER. Anaphylaxis. J Allergy Clin Immunol 2010;125: S161-81.

30. Sampson HA, Mendelson L, Rosen JP. Fatal and near-fatal anaphylactic reactions to food in children and adolescents. $N$ Engl J Med 1992;327:380-4.

31. Bock SA, Munoz-Furlong A, Sampson HA. Fatalities due to anaphylactic reactions to foods. J Allergy Clin Immunol 2001;107:191-3.

32. Fleming JT, Clark S, Camargo CA Jr, et al. Early treatment of food-induced anaphylaxis with epinephrine is associated with a lower risk of hospitalization. J Allergy Clin Immunol Pract 2015;3:57-62.

33. Simons FE, Clark S, Camargo CA Jr. Anaphylaxis in the community: learning from the survivors. J Allergy Clin Immunol 2009;124:301-6.
34. Sicherer SH, Simons FE; Section on Allergy and Immunology, American Academy of Pediatrics. Self-injectable epinephrine for first-aid management of anaphylaxis. Pediatrics 2007;119:638-46.

35. Campbell RL, Bellolio MF, Knutson BD, et al. Epinephrine in anaphylaxis: higher risk of cardiovascular complications and overdose after administration of intravenous bolus epinephrine compared with intramuscular epinephrine. J Allergy Clin Immunol Pract 2015;3:76-80.

36. Sicherer SH. Clinical implications of cross-reactive food allergens. J Allergy Clin Immunol 2001;108:881-90.

37. Savage JH, Matsui EC, Skripak JM, et al. The natural history of egg allergy. J Allergy Clin Immunol 2007;120:1413-7.

38. Sicherer SH, Wood RA, Vickery BP, et al. The natural history of egg allergy in an observational cohort. J Allergy Clin Immunol 2014;133:492-9.

39. Wood RA, Sicherer SH, Vickery BP, et al. The natural history of milk allergy in an observational cohort. J Allergy Clin Immunol 2013;131:805-12.

40. Skripak JM, Matsui EC, Mudd K, et al. The natural history of IgE-mediated cow's milk allergy. J Allergy Clin Immunol 2007; 120:1172-7

41. Savage JH, Kaeding AJ, Matsui EC, et al. The natural history of soy allergy. J Allergy Clin Immunol 2010;125:683-6.

42. Keet CA, Matsui EC, Dhillon G, et al. The natural history of wheat allergy. Ann Allergy Asthma Immunol 2009;102:410-5.

43. Skolnick HS, Conover-Walker MK, Koerner CB, et al. The natural history of peanut allergy. J Allergy Clin Immunol 2001;107: 367-74.

44. Ho MH, Wong WH, Heine RG, et al. Early clinical predictors of remission of peanut allergy in children. J Allergy Clin Immunol 2008;121:731-6.

45. Peters RL, Allen KJ, Dharmage SC, et al. Natural history of peanut allergy and predictors of resolution in the first 4 years of life: a population-based assessment. J Allergy Clin Immunol 2015; 135:1257-66.e1-2.

46. Fleischer DM, Conover-Walker MK, Matsui EC, et al. The natural history of tree nut allergy. J Allergy Clin Immunol 2005; 116:1087-93.

47. Kelso JM, Greenhawt MJ, Li JT, et al. Adverse reactions to vaccines practice parameter 2012 update. J Allergy Clin Immunol 2012;130:25-43

48. Canadian Immunization Guide. Ottawa: Public Health Agency of Canada; 2014. Available: www.phac-aspc.gc.ca/publicat/ cig-gci/index-eng.php (accessed 2015 Nov. 21).

49. Kelso JM. Administering influenza vaccine to egg-allergic persons. Expert Rev Vaccines 2014;13:1049-57.

50. Freigang B, Jadavji TP, Freigang DW. Lack of adverse reactions to measles, mumps, and rubella vaccine in egg-allergic children. Ann Allergy 1994;73:486-8.

51. James JM, Burks AW, Roberson PK, et al. Safe administration of the measles vaccine in children allergic to eggs. $N$ Engl J Med 1995;332:1262-6.

52. Moore DL. Vaccine recommendations for children and youth for the 2015/2016 influenza season. Paediatr Child Health 2015 20:389-94.

53. Turner PJ, Southern J, Andrews NJ. Safety of live attenuated influenza vaccine in young people with egg allergy: multicentre prospective cohort study. BMJ 2015;351:h6291.

54. Rutkowski K, Ewan PW, Nasser SM. Administration of yellow fever vaccine in patients with egg allergy. Int Arch Allergy Immunol 2013;161:274-8.

Affiliations: Division of Paediatric Allergy and Clinical Immunology (Abrams), University of Manitoba, Winnipeg, Man.; Division of Allergy and Immunology (Sicherer), Department of Pediatrics, Icahn School of Medicine at Mount Sinai, Jaffe Food Allergy Institute, New York, NY

Contributors: Both authors contributed substantially to the conception and design of the review. Elissa Abrams drafted the initial work, and Scott Sicherer critically revised it. Both authors approved the final version to be published and agreed to act as guarantors of the work.

Acknowledgement: The authors thank Drs. Alex Singer and Teresa Cavett for their review of the manuscript. 